\title{
Predisposition to Obesity: Should We Target Those Most Susceptible?
}

\author{
Nanna Julie Olsen • Erik Lykke Mortensen • \\ Berit Lilienthal Heitmann
}

Published online: 25 January 2012

(C) The Author(s) 2012. This article is published with open access at Springerlink.com

\begin{abstract}
Obesity prevention should remain a priority, even if there is some suggestion that the epidemic may presently have reached a stable level. However, previous interventions have not been effective in preventing overweight and obesity, and at the same time studies suggest that some subgroups are more predisposed to future obesity. The purpose of this paper is to review interventions on obesity prevention published during the past year, and to examine if interventions targeting predisposed groups or individuals seem more efficient in preventing obesity than studies targeting general populations. Among 15 identified studies, 7 targeted predisposed children or
\end{abstract}

N. J. Olsen $(\bowtie)$

Research Unit for Dietary Studies,

Institute of Preventive Medicine,

Copenhagen Capital Region, Copenhagen University Hospitals,

Øster Søgade 18.1,

Copenhagen, Denmark

e-mail: njo@ipm.regionh.dk

\section{E. L. Mortensen}

Department of Public Health and Center for Healthy Aging, University of Copenhagen,

Øster Farimagsgade 5, Building 15,

Copenhagen, Denmark

e-mail: elme@sund.ku.dk

\section{B. L. Heitmann}

National Institute of Public Health,

University of Southern Denmark,

Copenhagen, Denmark

e-mail: blh@ipm.regionh.dk

\section{B. L. Heitmann}

Institute of Preventive Medicine,

Copenhagen Capital Region, Copenhagen University Hospitals, Copenhagen, Denmark adolescents. More of the studies targeting predisposed individuals were able to show significant effects than the studies targeting general populations. Most studies targeting predisposed defined the predisposition based on ethnicity or socioeconomic status. Thus, we may be more successful in preventing obesity when targeting predisposed individuals, but more studies are needed before a firm conclusion can be drawn.

Keywords Obesity · Prevention · Susceptibility · Predisposition

\section{Introduction}

The increased prevalence of obesity is causing a great need for effective intervention programs [1]. Even though a possible leveling off of the obesity epidemic has been observed, prevention of obesity should remain a priority, as previous periods of stability in the epidemic have generally been followed by further increases [2•]. Numerous controlled primary intervention studies among children and adolescents have been conducted during the past 20 to 30 years, the majority of which were unable to prevent excessive weight gain. A Cochrane review from 2005 suggested that less than $20 \%$ of the interventions on children published between 1990 and 2005 showed an effect and further suggested that a focus on short-term behavior change was unlikely to have a sustained impact on the weight status of children [3]. The review concluded that the current evidence suggests that many diet and exercise interventions are in fact not effective in preventing excess weight gain in children, despite being apparently able to change diet and physical activity behavior in a more healthy direction. In this regard, 
the general lack of success in preventive interventions may relate to the fact that obesity is not simply an environmental cause, but is also under the influence of genes and pre- and perinatal factors [4]. It has been shown that some subgroups are at a particularly high risk of becoming obese, based on biological factors such as obesity among first-degree relatives and high birth weight [5], or based on cultural factors such as socioeconomic status or ethnicity [6•]. In the face of the general lack of successes in the previous interventions, it may be suggested that a target of those subgroups who are particular susceptible to overweight and obesity may prove to be a more effective intervention strategy than targeting general populations, where intervention effects confined to high-risk individuals may be diluted. In this regard, predisposition to obesity may be defined as individual, preexisting differences in certain characteristics that are associated with increased likelihood of excess weight gain from normal weight to overweight and obesity [7].

The purpose of the present paper was to review results from recent obesity intervention preventions published during the past year, and to examine if studies targeting highrisk groups or individuals seem more successful in relation to preventing overweight and obesity than studies targeting general population groups.

\section{Materials and Methods}

A literature search was conducted in the Medline database, using the keywords "obesity prevention," "obesity intervention," "obesity predisposition," "obesity susceptibility," and "prevention high-risk obesity" and filtering studies conducted on humans and published in English during the last year (September 2010-September 2011). Only intervention studies were included.

Inclusion criteria were interventions targeting prevention of overweight or obesity, applying one or more anthropometric measurements as outcome and reporting the effects of the interventions on anthropometric measurements. Exclusion criteria were interventions targeting treatment of overweight or obesity, not applying anthropometric measurements as outcome, targeting non-healthy individuals, study protocols, and studies reporting other aspects of the intervention than the effects on anthropometric measurements.

All studies included in the search were reviewed for the setting of the intervention, the sample size, fraction of normal weight and overweight subjects at baseline and if the effects of the intervention for the normal- and the overweight subjects were reported separately. Furthermore, the studies were reviewed for whether the intervention targeted one or more groups at high risk of developing overweight, if the study population consisted of infants ( $0-2$ years), children (312 years), or adolescents (13-19 years), and the size of the effect of the intervention. Finally, those studies that targeted one or more groups at high risk of developing overweight were further examined to determine if the high risk was biological or cultural.

\section{Results}

A total of 15 studies met the inclusion criteria (Table 1).

\section{Setting}

Of the 15 studies included, 8 interventions were conducted in a school setting $[8-11,12 \cdot, 13-15]$. One study was conducted in a kindergarten setting [16], one study was conducted in a home setting [17], and three studies were conducted in health centers or clinics [18-20]. Two studies compared interventions with two different settings, a community versus a community and family-based intervention [21] and a community center and family-based intervention versus an individual intervention [22]. These two studies were consequently not able to compare the effects of the intervention to a control group. One study did not include a control group [11]. One study compared two different interventions, one with less intense physical activity and one with aerobic exercises, and found a significant effect of the prevalence of overweight and obesity in both groups, but no significant difference between the groups [8]. Effects did not appear to be systematically dependent on the setting in which the intervention was conducted (Table 1).

\section{Participants}

All studies were conducted in infants, children, or adolescents. None of these study populations consisted of only normal weight individuals, but included both normal weight and overweight/obese in various fractions (Table 1). Eleven studies reported the baseline fraction of normal weight or overweight participants [8-11, 12•, $14-16,19,21,22]$, whereas in the remaining four studies this information was not available. One intervention was conducted in pregnant women only 4 weeks before expected delivery, and was consequently unable to report the baseline distribution of normal weight and overweight [20].

\section{Outcome}

Most studies examined effects of the intervention in relation to changes in body mass index (BMI) or BMI z-scores (Table 1). Only two studies reported a change in percentage body fat; here, one study reported an 
Table 1 Intervention studies according to age group, $n$, setting, baseline fraction of normal weight and overweight participants, targeting high-risk groups and significant effect of the intervention

\begin{tabular}{|c|c|c|c|c|c|c|}
\hline Study & Age group & $n$ & Setting & $\begin{array}{l}\text { Baseline fraction } \\
\text { normal weight/ } \\
\text { overweight } \\
\text { (including obese) }\end{array}$ & $\begin{array}{l}\text { Targeting } \\
\text { high-risk } \\
\text { groups }\end{array}$ & $\begin{array}{l}\text { Significant effect of the } \\
\text { intervention }\end{array}$ \\
\hline Karanja et al. [21] & Infants & 205 & $\begin{array}{l}\text { Community vs community } \\
\text { and family-based }\end{array}$ & $61 \% / 39 \%$ & Yes & $\begin{array}{l}+^{\mathrm{a}}(\mathrm{BMI} z \text {-score, community and } \\
\text { family-based intervention received })\end{array}$ \\
\hline Paul et al. [17] & Infants & 160 & $\begin{array}{l}\text { Home ( } 2 \text { different interventions, } \\
\text { reception of none, one, or } \\
\text { both interventions) }\end{array}$ & Not reported & No & $\begin{array}{c}+{ }^{\mathrm{b}} \text { (Weight-for-length percentile, } \\
\text { both interventions received) }\end{array}$ \\
\hline Robinson et al. [22] & Children & 261 & $\begin{array}{l}\text { Community centers and } \\
\text { family-based vs individual }\end{array}$ & $49 \% / 51 \%$ & Yes & - (BMI) \\
\hline Wake et al. [18] & Infants & 328 & Well-child centers & Not reported & No & $\begin{array}{c}\text { - (BMI z-score, percentage } \\
\text { overweight/obese, WC) }\end{array}$ \\
\hline Nemet et al. [16] & Children & 725 & Kindergarten & $72 \% / 28 \%$ & Yes & $\begin{array}{l}+^{\mathrm{a}} \text { (Number of overweight) } \\
-(\mathrm{BMI})\end{array}$ \\
\hline $\begin{array}{l}\text { Balas-Nakash et } \\
\text { al. [8] }\end{array}$ & Children & 319 & $\begin{array}{l}\text { School (2 different } \\
\text { interventions compared) }\end{array}$ & $54 \% / 46 \%$ & No & - (BMI, body fat) \\
\hline Foster et al. [9] & Children & 4603 & School & $50 \% / 50 \%$ & Yes & $\begin{array}{l}+^{\mathrm{a}}(\text { BMI z-score }) \\
+^{\mathrm{a}}(\text { Prevalence obesity) } \\
-(\text { Prevalence overweight/obesity) }\end{array}$ \\
\hline Tucker et al. [10] & Children & 99 & School & $72 \% / 27 \%$ & No & - (BMI, BMI percentile) \\
\hline Hawthorne et al. [11] & Children & 1074 & School & $55 \% / 45 \%$ & Yes & - (BMI, WC) \\
\hline Lubans et al. [12•] & Adolescents & 100 & School & $65 \% / 35 \%$ & Yes & $\begin{array}{l}+^{\mathrm{b}}(\mathrm{BMI}) \\
+^{\mathrm{b}}(\text { BMI z-score }) \\
+^{\mathrm{a}}(\text { Body fat }) \\
-(\mathrm{WC})\end{array}$ \\
\hline Hoffman et al. [13] & Children & 297 & School & Not reported & No & - (BMI) \\
\hline Klesges et al. [19] & Children & 303 & $\begin{array}{l}\text { Community centers and } \\
\text { YMCAs }\end{array}$ & $43 \% / 57 \%$ & Yes & - (BMI) \\
\hline Luoto et al. [20] & Infants & 159 & Antenatal clinics & Not reported & No & - (BMI) \\
\hline $\begin{array}{l}\text { Neumark-Sztainer } \\
\text { et al. [14] }\end{array}$ & Adolescents & 356 & School & $54 \% / 46 \%$ & No & - (BMI, body fat) \\
\hline Hendy et al. [15] & Children & 382 & School & $65 \% / 35 \%$ & No & - (BMI percentile) \\
\hline
\end{tabular}

${ }^{\mathrm{a}} P \leq 0.05$

${ }^{\mathrm{b}} P \leq 0.01$

$B M I$ body mass index, $W C$ waist circumference

effect [12•], whereas the other did not find an effect of the intervention [14]. Thus, the results of the reviewed intervention studies did not seem to depend systematically on the choice of the anthropometric outcome measure (Table 1).

\section{Intervention Effects}

A total of five studies found a significant positive effect of the intervention $[9,12 \bullet, 16,17,21]$. Two studies showed an effect on the prevalence of overweight/obesity, whereas three studies showed an effect on BMI or BMI z-score (Table 1). Of the five studies that were able to show a significant effect of the intervention, four studies targeted one or more groups at high risk of developing overweight $[9,12 \bullet, 16,21]$.

\section{High-Risk Samples}

Eight studies did not target high-risk groups (Table 1) and among these, only one study showed a significant positive effect of the intervention [17]. Seven studies targeted one or more high-risk groups $[9,11,12 \cdot 16,19,21,22]$. Of these seven studies, four were able to show a significant effect of the intervention (Table 1).

\section{Defining High Risk}

Table 2 shows the characteristics and effects of the interventions that targeted high-risk groups. Five of seven studies defined high risk based on ethnicity and/or socioeconomic status $[9,11,12 \cdot 16,21]$. One study identified high-risk 
Table 2 Interventions targeting high-risk groups according to exact risk factor, cultural/biological risk factors used to classify "high risk," and significant effect of the intervention

\begin{tabular}{|c|c|c|c|c|}
\hline Study & Setting & $\begin{array}{l}\text { Exact risk factors used to } \\
\text { classify "high risk" }\end{array}$ & $\begin{array}{l}\text { Cultural/biological } \\
\text { risk factors used to } \\
\text { classify "high risk" }\end{array}$ & Significant effect of the intervention \\
\hline Karanja et al. [21] & $\begin{array}{l}\text { Community vs community and } \\
\text { family-based }\end{array}$ & Ethnicity & Cultural & $\begin{array}{l}+^{\mathrm{a}}(\mathrm{BMI} z \text {-score, community and } \\
\text { family-based intervention received) }\end{array}$ \\
\hline Robinson et al. [22] & $\begin{array}{l}\text { Community centers and family- } \\
\text { based vs individual }\end{array}$ & $\begin{array}{l}\text { Ethnicity } \\
\text { Socioeconomic status } \\
\text { Parental overweight }\end{array}$ & $\begin{array}{l}\text { Cultural } \\
\text { Cultural } \\
\text { Biological }\end{array}$ & - (BMI) \\
\hline Nemet et al. [16] & Kindergarten & Socioeconomic status & Cultural & $\begin{array}{l}+^{\mathrm{a}} \text { (Number of overweight) } \\
-(\mathrm{BMI})\end{array}$ \\
\hline Foster et al. [9] & School & $\begin{array}{l}\text { Ethnicity } \\
\text { Socioeconomic status }\end{array}$ & $\begin{array}{l}\text { Cultural } \\
\text { Cultural }\end{array}$ & $\begin{array}{l}+^{\mathrm{a}}(\text { BMI z-score }) \\
+^{\mathrm{a}}(\text { Prevalence obesity }) \\
-(\text { Prevalence overweight/obesity })\end{array}$ \\
\hline Hawthorne et al. [11] & School & $\begin{array}{l}\text { Ethnicity } \\
\text { Socioeconomic status }\end{array}$ & $\begin{array}{l}\text { Cultural } \\
\text { Cultural }\end{array}$ & - (BMI, WC) \\
\hline Lubans et al. [12•] & School & Socioeconomic status & Cultural & $\begin{array}{l}+^{\mathrm{b}}(\mathrm{BMI}) \\
+^{\mathrm{b}}(\text { BMI z-score }) \\
+^{\mathrm{a}}(\text { Body fat }) \\
-(\mathrm{WC})\end{array}$ \\
\hline Klesges et al. [19] & Community centers and YMCAs & $\begin{array}{l}\text { Ethnicity } \\
\text { Parental overweight }\end{array}$ & $\begin{array}{l}\text { Cultural } \\
\text { Biological }\end{array}$ & - (BMI) \\
\hline
\end{tabular}

${ }^{\text {a } P \leq 0.05}$

${ }^{\mathrm{b}} P \leq 0.01$

$B M I$ body mass index, $W C$ waist circumference

individuals based on ethnicity or parental overweight [19], and one study identified predisposed individuals based on ethnicity, low income, and parental overweight [22]. However, there was no information on the proportion of participants selected based on each of the risk factors. The success of highrisk interventions did not appear to be dependent on the setting of the intervention (Table 2).

\section{Discussion}

In the studies published on prevention of overweight and obesity over the past year, nearly half targeted groups or individuals susceptible to future overweight or obesity. Intervention studies that did not target high-risk groups generally were unable to show an effect of the intervention (Table 1). This may reflect that most intervention effects were not sufficiently large to induce significant overall change, and it is probable that effects may have been observed, if only the subgroup of individuals with a predisposition to obesity had been included. This notion is substantiated by the finding that more than half of the studies targeting high-risk groups showed significant positive results compared with only 1 in 8 of the studies targeting non-selected groups.
We found that interventions targeting high-risk groups and conducted in school settings appeared to be slightly more effective than studies conducted in community settings (Table 2). However, it may be argued that when high-risk individuals are selected on group level (eg, schools in a low socioeconomic area), clearly there will be a variation in the susceptibility between individuals, and thus not all individuals included may in fact be susceptible to overweight and obesity, which may have caused inflation of the results.

As suggested by Sørensen [7], there may be a distinction between predisposition, operating unmodified by the normally occurring environmental variation, and susceptibility, which is modifiable predisposition. When planning prevention studies it may therefore be important to consider which risk factors reflect modifiable predisposition or susceptibility to obesity [7]. Thus, it may be hypothesized that different interventions have different effects depending on whether the study population is predisposed or susceptible to obesity. As far as we know, interventions targeting high-risk individuals have hitherto not been evaluated in different target populations, but ideally the efficiency of interventions should be evaluated in study populations selected on the basis of different risk factors. This may alleviate identifying more precisely which risk factors reflect unmodifiable 
predisposition and which risk factors reflect susceptibility, and may in a long-term perspective make it possible to identify groups or individuals based on the most efficient risk factors to intervene against.

Among the reviewed studies, the vast majority targeted predisposed groups or individuals at high risk due to low socioeconomic status or ethnicity, whereas only two studies included parental overweight (Table 2). In this context, it may be discussed if ethnicity is a biological or a cultural risk factor. Ethnicity may be classified as a biological risk factor, with the susceptibility for future overweight and obesity being expressed as variations in genetic predisposition to overweight and obesity [23]. Conversely, ethnicity may also be classified as a cultural risk factor, being a marker of (low) socioeconomic status and in some cases lifestyle and behavior associated with overweight and obesity. Consequently, the impact of ethnicity on susceptibility to overweight and obesity may vary according to country or culture, since social status and lifestyle related to ethnicity may differ between countries and cultures. In addition, it may not be completely unproblematic to classify ethnicity, as some individuals may have bi-ethnic parents or grandparents.

Similar concerns may be relevant to consider in relation to low socioeconomic status. Thus, it may be argued that low socioeconomic status is not purely a cultural factor, but can also be considered a marker of biological risk (eg, overweight in first-degree relatives). Thus, the objectives for selecting a specific group of predisposed individuals should be considered thoroughly prior to planning of the intervention.

The available studies do not answer the question on how to best define high-risk status or how the definition of high-risk status may interact with the nature of interventions. If the risk of future overweight and obesity approximate a normal distribution in the population, a relatively small number of individuals may be at substantial risk, and this raises the issue of whether the efficacy of interventions is primarily related to absolute risk or relative risk. If the population distribution of risk is positively skewed, it will be easier to use a definition of high risk, which includes a large number of individuals with both substantial relative and absolute high risk of developing overweight and obesity. Irrespective of the definition of high risk, some individuals will be at higher risk than others (different levels of susceptibility), and consequently future studies should evaluate interventions in samples of different levels of susceptibility.

Furthermore, the effects of different interventions may not only depend on the quantitative risk, but also on the factors leading to increased risk. Thus, preventing obesity in individuals with biological predisposition may require different approaches than in individuals with cultural predisposition. Biological susceptibility may not only require different lifestyle and behavioral changes than social and cultural susceptibility, but it may also be hypothesized to have a weaker association with socioeconomic status, and may therefore have a different starting point and need different or even shorter interventions. Moreover, there may be a difference in the level of motivation and the required motivation techniques between individuals predisposed based on either biological or cultural factors. However, investigating differences between biological and cultural susceptibility in relation to interventions may be challenging, due to the previously described complexity in distinguishing between biological or cultural risk factors. One way to overcome this challenge may be to conduct studies comparing interventions in samples with clearly defined biological susceptibility (eg, high birth weight) or clearly defined cultural factors (eg, children with both parents being the same ethnicity).

The studies included in this review were performed in children or adolescents. The lack of interventions aiming at preventing obesity among adults is striking, but may depend on the increased focus on childhood obesity in general, or be the result of the recent literature concluding that prevention should start as early as possible [24].

None of the reviewed studies examined effects of intervening according to individual needs and resources rather than an intervention "package" delivered to all participants. Thus, interventions targeting at an individual level are needed in future studies. It may be speculated that the level of predisposition to future overweight and obesity varies according to what risk factor is selected for defining the predisposition. As the vast majority of the reviewed interventions targeting predisposed individuals considered socioeconomic status or ethnicity, more studies are needed that select individuals based on biological risk factors, such as high birth weight, low birth weight or early catch-up growth. This has also been suggested by a recent report of action by the Danish Board of Technology, concluding that "in order to curb the obesity epidemic, ....there is a need for improving the effect of obesity prevention in those groups who are in the highest risk of overweight and obesity" [25]. The report outlined specific obesity prevention intervention projects that targeted explicit high-risk groups and gave suggestions as to how such efforts could be implemented in existing settings and arenas.

Compared with the results from many of the earlier studies and reviews $[3,26]$, it seems that an increasing number of the more recent studies were able to show an effect. This may reflect improved interventions in general, publication bias, or as suggested here, that the more recent studies to a higher degree than previously reported focused on targeting high-risk groups in obesity prevention.

Since none of the studies in the present review were focusing on normal weight predisposed individuals only, none of 
them can be considered primary prevention interventions of overweight and obesity. In fact, only one study examined the intervention effects separately for normal weight and overweight participants and that study found that both among the normal weight and overweight children there was a decline in BMI percentile after a 3-month intervention. They also found that the children who were overweight at baseline regained weight 6 months later [15]. Thus, whether the effects reported from most of the included studies were consequences of treating obesity instead of preventing it cannot be evaluated. In the search for effective tools for obesity prevention, it may be of high relevance to distinguish between primary prevention, where weight development among those normal weight subjects is considered, and secondary prevention, where overweight is treated or prevented from developing further, as the same type of interventions may not have the same effect for the two types of prevention interventions.

In summary, the literature included in this review suggests that targeting susceptible groups or individuals in obesity prevention may be a more effective way to prevent obesity than to target general population samples. However, it may be argued that even if there is still only few targeted interventions, this strategy may be preferable. Susceptible individuals may be selected based on phenotypical rather than genotypical characteristics, as the observed effects on future obesity development from recently identified genetic loci are small [27]. Only a few percentage of the variance in BMI can be explained by obesity susceptibility loci variants, whereas genetic factors in general has been shown to explain between $40 \%$ and $70 \%$ of the variation in common obesity in family and twin studies [27]. Consequently, at present, it may be a more efficient strategy to select intervention groups based on predisposing factors, which have been documented to explain a substantial part of the variance in obesity (ex, individuals with obesity among their first-degree relatives).

\section{Conclusions}

Interventions targeting high-risk groups may be more effective in preventing overweight and obesity compared with interventions targeting general population groups. However, most of the previous studies that used high-risk strategies to identify susceptibility to future overweight and obesity, were selecting high risk individuals based on their ethnicity or socioeconomic status only, and more studies targeting individuals with for example a high birth weight or an early catch-up growth are needed.

Disclosure No potential conflicts of interest relevant to this article were reported.
Open Access This article is distributed under the terms of the Creative Commons Attribution Noncommercial License which permits any noncommercial use, distribution, and reproduction in any medium, provided the original author(s) and source are credited.

\section{References}

Papers of particular interest, published recently, have been highlighted as:

- Of importance

1. World Health Organization. Obesity: preventing and managing the global epidemic. Report of a WHO consultation on Obesity. 2000, 840. WHO Technical Report Series.

2. Rokholm B, Baker JL, Sorensen TI. The levelling off of the obesity epidemic since the year 1999-a review of evidence and perspectives. Obes Rev. 2010;11:835-46. This study found that even though the obesity epidemic may be taking a break, previous stable phases have been followed by further increases. Therefore, obesity prevention should remain a priority.

3. Summerbell CD, Waters E, Edmunds LD, et al. Interventions for preventing obesity in children. Cochrane Database Syst Rev. 2005; CD001871.

4. Newell A, Zlot A, Silvey K, Arail K. Addressing the obesity epidemic: a genomics perspective. Prev Chronic Dis. 2007;4:A31.

5. Danielzik S, Czerwinski-Mast M, Langnase K, et al. Parental overweight, socioeconomic status and high birth weight are the major determinants of overweight and obesity in 5-7 y-old children: baseline data of the Kiel Obesity Prevention Study (KOPS). Int J Obes Relat Metab Disord. 2004;28:1494-502.

6. - Deboer MD. Ethnicity, obesity and the metabolic syndrome: implications on assessing risk and targeting intervention. Expert Rev Endocrinol Metab. 2011;6:279-89. This study reviews ethnicity and obesity in children, and brings attention to the applicability of ethnicity as a determinant for predisposition to overweight and obesity.

7. Sørensen TIA. Challenges in the study of causation of obesity. Proc Nutr Soc. 2009;68:43-54.

8. Balas-Nakash M, Itez-Arciniega A, Perichart-Perera O, et al. The effect of exercise on cardiovascular risk markers in Mexican school-aged children: comparison between two structured group routines. Salud Publica Mex. 2010;52:398-405.

9. Foster GD, Linder B, Baranowski T, et al. A school-based intervention for diabetes risk reduction. N Engl J Med. 2010;363:44353.

10. Tucker S, Lanningham-Foster L, Murphy J, et al. A school based community partnership for promoting healthy habits for life. J Community Health. 2011;36:414-22.

11. Hawthorne A, Shaibi G, Gance-Cleveland B, McFall S. Grand Canyon Trekkers: school-based lunchtime walking program. J Sch Nurs. 2011;27:43-50.

12. - Lubans DR, Morgan PJ, Aguiar EJ, Callister R. Randomized controlled trial of the Physical Activity Leaders (PALs) program for adolescent boys from disadvantaged secondary schools. Prev Med. 2011;52:239-46. This study targeted individuals predisposed to future overweight and obesity and found the strongest effect of the intervention on BMI, BMI z-scores, and body fat.

13. Hoffman JA, Thompson DR, Franko DL, et al. Decaying behavioral effects in a randomized, multi-year fruit and vegetable intake intervention. Prev Med. 2011;52:370-5.

14. Neumark-Sztainer DR, Friend SE, Flattum CF, et al. New movespreventing weight-related problems in adolescent girls a grouprandomized study. Am J Prev Med. 2010;39:421-32. 
15. Hendy HM, Williams KE, Camise TS. Kid's Choice Program improves weight management behaviors and weight status in school children. Appetite. 2011;56:484-94.

16. Nemet D, Geva D, Eliakim A. Health promotion intervention in low socioeconomic kindergarten children. J Pediatr. 2011;158:796-801.

17. Paul IM, Savage JS, Anzman SL, et al. Preventing obesity during infancy: a pilot study. Obesity (Silver Spring). 2011;19:353-61.

18. Wake M, Price A, Clifford S, et al. Does an intervention that improves infant sleep also improve overweight at age 6? Followup of a randomised trial. Arch Dis Child. 2011;96:526-32.

19. Klesges RC, Obarzanek E, Kumanyika S, et al. The Memphis Girls' health Enrichment Multi-site Studies (GEMS): an evaluation of the efficacy of a 2-year obesity prevention program in African American girls. Arch Pediatr Adolesc Med. 2010;164:100714.

20. Luoto R, Kalliomaki M, Laitinen K, Isolauri E. The impact of perinatal probiotic intervention on the development of overweight and obesity: follow-up study from birth to 10 years. Int J Obes (Lond). 2010;34:1531-7.

21. Karanja N, Lutz T, Ritenbaugh C, et al. The TOTS community intervention to prevent overweight in American Indian toddlers beginning at birth: a feasibility and efficacy study. J Community Health. 2010;35:667-75.

22. Robinson TN, Matheson DM, Kraemer HC, et al. A randomized controlled trial of culturally tailored dance and reducing screen time to prevent weight gain in low-income African American girls: Stanford GEMS. Arch Pediatr Adolesc Med. 2010;164:9951004.

23. Bressler J, Kao WH, Pankow JS, Boerwinkle E. Risk of type 2 diabetes and obesity is differentially associated with variation in FTO in whites and African-Americans in the ARIC study. PLoS One. 2010;5:e10521.

24. Rugholm S, Baker JL, Olsen LW, et al. Stability of the association between birth weight and childhood overweight during the development of the obesity epidemic. Obes Res. 2005;13:2187-94.

25 . The Danish Board of Technology. Targeted prevention of obesity more effect for the money. 2011.

26. Monasta L, Batty GD, Macaluso A, et al. Interventions for the prevention of overweight and obesity in preschool children: a systematic review of randomized controlled trials. Obes Rev. 2011;12:e107-18.

27. Loos RJ. Recent progress in the genetics of common obesity. $\mathrm{Br} \mathrm{J}$ Clin Pharmacol. 2009;68:811-29. 